\title{
RADIAL GROWTH OF HARMONIC FUNCTIONS IN THE UNIT BALL
}

\author{
KJERSTI SOLBERG EIKREM and EUGENIA MALINNIKOVA*
}

\begin{abstract}
Let $\Psi_{v}$ be the class of harmonic functions in the unit disk or unit ball in $\mathrm{R}^{m}$ which admit a radial majorant $v(r)$. We prove that a function in $\Psi_{v}$ may grow or decay as fast as $v$ only along a set of radii of measure zero. For the case when $v$ fulfills a doubling condition, we give precise estimates of these exceptional sets in terms of Hausdorff measures.
\end{abstract}

\section{Introduction}

Radial behavior of harmonic functions in the unit disk and unit ball in $\mathrm{R}^{m}$ is a classical topic in analysis. In this article we consider harmonic functions bounded a priori by some radial majorant and discuss their radial growth.

It follows from a theorem of N. N. Lusin and I. I. Privalov, see [15], that there exist harmonic functions in the unit disk that tend to infinity along almost each radius. Moreover, a generalization of this result obtained by J.-P. Kahane and Y. Katznelson [11], shows that such functions may be bounded by an arbitrarily slow growing radial majorant.

Let $v(r)$ be a positive increasing continuous function on $[0,1)$ and assume that $\lim _{r \rightarrow 1} v(r)=+\infty$. Let $\mathrm{B}$ be the unit ball in $\mathrm{R}^{m}$, we define

$$
\Phi_{v}^{m}=\{u: \mathrm{B} \rightarrow \mathrm{R}, \Delta u=0, u(x) \leq K v(|x|) \text { for some } K>0\},
$$

and

$$
\Psi_{v}^{m}=\{u: \mathrm{B} \rightarrow \mathrm{R}, \Delta u=0,|u(x)| \leq K v(|x|) \text { for some } K>0\} .
$$

Harmonic functions of the class $\Phi_{v}^{2}$ with $v(r)=|\log (1-r)|$ were studied by B. Korenblum in [12]. This class as well as more general classes that correspond to $v(r)=|\log (1-r)|^{s}$ appear in connection with the related spaces of analytic functions in the unit disk, see also [16], [3]. Two-sided estimates and classes $\Psi_{v}^{2}$ arise when one considers invertible elements in these spaces of analytic functions as in [2]. Radial growth of harmonic functions in the unit

\footnotetext{
* The second author was supported by the Research Council of Norway, grant 185359/V30.
}

Received 18 October 2010. 
disk bounded by a multiple of $|\log (1-r)|$ was studied by A. Borichev, Yu. Lyubarskii, E. Malinnikova and P. Thomas in [4], see also [13]. In this article we discuss to what extent the results in [4] remain true for general majorants and higher dimensional spaces.

The main aim of this work is to estimate the size of the set of the radii along which a function from $\Phi_{v}^{m}$ or $\Psi_{v}^{m}$ grows or decays as fast as the majorant $v(r)$. For each function $u \in \Phi_{v}^{m}$ we define the following subsets of the unit sphere

$$
\begin{aligned}
& E^{-}(u)=\left\{y \in S: \limsup _{r \rightarrow 1-} \frac{u(r y)}{v(r)}<0\right\}, \\
& E^{+}(u)=\left\{y \in S: \liminf _{r \rightarrow 1-} \frac{u(r y)}{v(r)}>0\right\} .
\end{aligned}
$$

Our first result is the following:

THEOREM 1.1. If $u \in \Psi_{v}^{m}$ then $\sigma\left(E^{-}(u)\right)=\sigma\left(E^{+}(u)\right)=0$, where $\sigma$ is the usual surface measure on the unit sphere in $\mathrm{R}^{m}$.

We give more precise estimates of the sets $E^{ \pm}(u)$ when $v$ satisfies the following doubling condition

$$
v(1-d / 2) \leq D v(1-d) .
$$

The constants $K$ and $D$ will preserve their identities throughout this article.

For every increasing continuous function $\lambda:[0,+\infty) \rightarrow[0,+\infty)$ with $\lambda(0)=0$ we denote by $\mathscr{H}_{\lambda}$ the corresponding Hausdorff measure.

THEOREM 1.2. Let $v$ satisfy (2).

(a) If $u \in \Phi_{v}^{m}$ and $\lambda$ is a continuous increasing function, $\lambda(0)=0$ and

$$
\lambda(t)=o\left(t^{m-1} v(1-t)^{\alpha}\right) \quad(t \rightarrow 0),
$$

for all $\alpha>0$, then $\mathscr{H}_{\lambda}\left(E^{+}(u)\right)=\mathscr{H}_{\lambda}\left(E^{-}(u)\right)=0$.

(b) For each $\beta>0$ there exists $u \in \Psi_{v}^{m}$ and an increasing continuous function $\lambda_{\beta}, \lambda_{\beta}(0)=0$ and $\lambda_{\beta}(t)=O\left(t^{m-1} v(1-t)^{\beta}\right)(t \rightarrow 0)$, such that $\mathscr{H}_{\lambda_{\beta}}\left(E^{+}(u)\right)>0$.

From (b) we see that the estimate for $E^{-}$in (a) is sharp as well, since $u \in \Psi_{v}^{m}$ and $E^{-}(-u)=E^{+}(u)$.

In this theorem there is no difference between the size of the sets $E^{ \pm}(u)$ for $u \in \Phi_{v}^{m}$ and $u \in \Psi_{v}^{m}$. The situation is different for positive harmonic functions as was also noted in [4]. We generalize the result on positive harmonic functions to a wide class of weights. 
THEOREM 1.3. Assume that $\lambda(t)=t^{m-1} v(1-t)$ is an increasing continuous function and $\lambda(0)=0$. There exists a positive function $u \in \Psi_{v}^{m}$ such that $\mathscr{H}_{\lambda}\left(E^{+}(u)\right)>0$.

As the next result shows, the choice of $\lambda$ for given $v$ in the theorem above is optimal. Moreover, no a priori growth estimate is needed for the estimate of the Hausdorff measure $\mathscr{H}_{\lambda}$ of the set of radii along which a positive harmonic function grows as $v(r)$. More precisely,

THeorem 1.4. Assume that $\lambda(t)=t^{m-1} v(1-t)$ is an increasing continuous function and $\lambda(0)=0$. Let $u$ be a positive harmonic function in the unit ball of $\mathrm{R}^{m}$, we define

$$
F_{v}^{+}(u)=\left\{y \in S: \limsup _{r \rightarrow 1-} \frac{u(r y)}{v(r)}>0\right\} .
$$

Then $F_{v}^{+}(u)$ is a countable union of sets of finite $\mathscr{H}_{\lambda}$-measure.

The article is organized as follows. We collect some preliminary results on harmonic measure and Hausdorff measures in the next section. Then we prove Theorem 1.1 and Theorem 1.2. For part (a) of Theorem 1.2 our arguments are similar to those in [4]. In higher dimensions they are based on estimates of harmonic measure due to B. E. Dahlberg, [6]. A new approach is used to construct examples of functions with a large set of extremal growth in dimension larger than two in the proof of Theorem 1.2(b).

In the last section we study the radial growth of positive harmonic functions. We prove Theorem 1.4 first, then we characterize boundary measures that correspond to positive functions in $\Psi_{v}^{m}$ and describe an example that proves Theorem 1.3. Both constructions (for Theorem 1.2(b) and Theorem 1.3) employ Cantor-type sets on the unit sphere.

\section{Preliminaries}

\subsection{Poisson kernel and some estimates}

Let $\sigma$ be the $(m-1)$-dimensional surface measure on $S$ and denote $\sigma(S)=$ $\gamma_{m-1}$. The Poisson kernel in the $m$-dimensional unit ball is

$$
P(x, \zeta)=\frac{1}{\gamma_{m-1}} \frac{1-|x|^{2}}{|x-\zeta|^{m}} .
$$

Assume for simplicity that $x=(1,0, \ldots, 0)$. Using hyperspherical coordinates for $\zeta \in S$ we have $\zeta=\left(\cos \phi, \zeta^{\prime}\right)$, where $\left|\zeta^{\prime}\right|=\sin \phi$. Let

$$
\tilde{P}_{m, r}(\phi)=\frac{1}{\gamma_{m-1}} \frac{1-r^{2}}{\left(1+r^{2}-2 r \cos \phi\right)^{m / 2}}
$$


and

$$
Q_{m}(r, \phi)=-\partial_{\phi} \tilde{P}_{m, r}(\phi)=\frac{1}{\gamma_{m-1}} \frac{m r\left(1-r^{2}\right) \sin \phi}{\left(1+r^{2}-2 r \cos \phi\right)^{(m+2) / 2}} .
$$

Then $P(r x, \zeta)=\tilde{P}_{m, r}(\phi)$.

Let $d(x, \zeta)$ be the geodesic distance between two points $x$ and $\zeta$ on $S$. Then let $B(x, \phi)=\{\zeta \in S: d(x, \zeta)<\phi\}$ be the hyperspherical cap of radius $\phi$ with center at $x$. It can be shown that for the $(m-1)$-dimensional surface measure of the cap

$$
C_{1} \phi^{m-1} \leq \sigma(B(x, \phi)) \leq C_{2} \phi^{m-1},
$$

where the constants depend on $m$.

We will need some estimates for integrals of $Q_{m}$.

(i) We have

$$
\begin{aligned}
\int_{0}^{1-r} Q_{m}(r, \phi) d \phi & \leq C_{3} \int_{0}^{1-r} \frac{r\left(1-r^{2}\right) \phi}{\left((1-r)^{2}+2 r(1-\cos \phi)\right)^{(m+2) / 2}} d \phi \\
& \leq C_{3} \int_{0}^{1-r} \frac{r\left(1-r^{2}\right) \phi}{(1-r)^{m+2}} d \phi,
\end{aligned}
$$

hence

$$
\int_{0}^{1-r} Q_{m}(r, \phi) d \phi \leq C_{4} \frac{1}{(1-r)^{m-1}} .
$$

(ii) For $d>0$

$$
\begin{aligned}
\int_{d}^{\pi} Q_{m}(r, \phi) d \phi & \leq C_{3} \int_{d}^{\pi} \frac{r\left(1-r^{2}\right) \phi}{\left((1-r)^{2}+r \frac{4}{\pi^{2}} \phi^{2}\right)^{m / 2+1}} d \phi \\
& \leq C_{3} \int_{d}^{\pi} \frac{r\left(1-r^{2}\right) \phi}{\left(r \frac{4}{\pi^{2}} \phi^{2}\right)^{m / 2+1}} d \phi,
\end{aligned}
$$

thus

$$
\int_{d}^{\pi} Q_{m}(r, \phi) d \phi \leq C_{5} r^{-m / 2} d^{-m} \leq C_{6} d^{-m}
$$

when $r>\frac{1}{2}$. 
(iii) Furthermore, by (4),

$$
\begin{aligned}
\int_{1-r}^{\pi} \sigma(B(x, \phi)) Q_{m}(r, \phi) d \phi & \leq \int_{1-r}^{\pi} C_{2} \phi^{m-1} Q_{m}(r, \phi) d \phi \\
& \leq C_{7} \int_{1-r}^{\pi} \frac{\left(1-r^{2}\right) \phi^{-2}}{r^{m / 2}} d \phi \\
& \leq C_{8} r^{-m / 2},
\end{aligned}
$$

so for $r>\frac{1}{2}$,

$$
\int_{1-r}^{\pi} \sigma(B(x, \phi)) Q_{m}(r, \phi) d \phi \leq C_{9}
$$

\subsection{Harmonic measure in Lipschitz domains}

A bounded domain $\Omega \in \mathrm{R}^{m}$ is a Lipschitz domain if there is a constant $C$ such that to each point $q \in \partial \Omega$ there corresponds a coordinate system $(\xi, \eta), \xi \in$ $\mathrm{R}^{m-1}, \eta \in \mathrm{R}$, and a function $\varphi$ such that $\left|\varphi\left(\xi_{1}\right)-\varphi\left(\xi_{2}\right)\right| \leq C\left|\eta_{1}-\eta_{2}\right|$ for which $\Omega \cap V=\{(\xi, \eta): \varphi(\xi)<\eta\}$ for some neighborhood $V$ of $q$. The smallest such constant $C$ is called the Lipschitz constant.

Let $S$ be the unit sphere in $\mathrm{R}^{m}$. For $\zeta \in S$ and $a<1$ we use the standard notation $\Gamma_{\zeta}^{a}=\operatorname{conv}(\zeta, a \mathrm{~B})$ for the convex hull of $\zeta$ and the $m$-dimensional ball of radius $a$. Given a compact set $F \in S$ we consider the cone-domain $G=G(F, a)=\bigcup_{\zeta \in F} \Gamma_{\zeta}^{a}$. It is a Lipschitz domain, and the Lipschitz constant of $G(F, a)$ depends on $a$ only. Given a Lipschitz domain $\Omega$, a subset $A \subset \partial \Omega$ and a point $z \in \Omega$, we denote by $\omega(z, A, \Omega)$ the harmonic measure of $A$ at the point $z$.

A celebrated result by B. E. Dahlberg [6] says that on the boundary of a Lipschitz domain the harmonic measure and the surface measure are mutually absolutely continuous. We need a quantitative form of this result for conedomains and refer the reader to [6], [10] and [1, Chapter 4.2].

Theorem A. Let $a>0$, then there exist $\alpha$ and $C$ that depend on $a$ and $m$ only such that for any cone-domain $G=G(F, a)$ in the unit ball of $\mathrm{R}^{m}$ and any $A \subset Q \subset \partial G$ the following inequality holds

$$
\frac{\omega(0, A, G)}{\omega(0, Q, G)} \geq C\left(\frac{\eta(A)}{\eta(Q)}\right)^{\alpha},
$$

where $Q$ is a ball on $\partial G$ and $\eta$ is the surface measure on $\partial G$. 


\subsection{Hausdorff measures}

We will refer to generalized Hausdorff measures in $\mathrm{R}^{m}$ as they are defined for example in [14, p. 59]. Let $h$ be an increasing continuous function on $[0,+\infty)$, $h(0)=0$, then for any $E \subset \mathrm{R}^{m}$

$$
\mathscr{H}_{h}(E)=\liminf _{\delta \rightarrow 0}\left\{\sum_{j} h\left(d_{j}\right): E \subset \bigcup_{j} F_{j}, d_{j}=\operatorname{diam}\left(F_{j}\right)<\delta\right\} .
$$

We assume in addition that $h(t / 2) \geq \operatorname{ch}(t)$ for some $c>0$. Then the Hausdorff measure is equivalent to the so-called net measure $N_{h}(E)$, defined with $F_{j}$ being half-open dyadic cubes with sides parallel to the coordinate axis, in the following sense: $\mathscr{H}_{h}(E) \leq N_{h}(E) \leq A(c, m) \mathscr{H}_{h}(E)$, see [14, p. 76]. Further, the following property holds, if $f: \mathrm{R}^{k} \rightarrow \mathrm{R}^{m}$ is a Lipschitz map and $E \subset \mathrm{R}^{k}$, then $\mathscr{H}_{h}(f(E)) \leq L \mathscr{H}_{h}(E)$, where $L$ depends on the Lipschitz constant of $f$ and on $c$. The proofs follow readily from the definitions.

We will use Cantor-type sets having the following structure:

$$
C=\bigcap_{s} C_{s}, \quad C_{s} \supset C_{s+1}, \quad C_{0}=[0,1],
$$

where each set $C_{s}$ is a union of $N_{s}$ segments $\left\{I_{j}^{(s)}\right\}_{j}$ of the same length $l_{s}$. For each such segment the intersection $C_{s+1} \cap I_{j}^{(s)}$ is a union of $k_{s}$ non-overlapping segments of length $l_{s+1}$. We assume, of course, that
(i) $l_{s} \searrow 0$ as $s \rightarrow \infty$,
(ii) $k_{s} l_{s+1}<l_{s}$,
and
(iii) $N_{s}=k_{0} k_{1} \ldots k_{s-1}$.

The next result is Theorem 3 in [4].

Lemma A. Let $\lambda:[0,1) \rightarrow[0,+\infty)$ be a continuous increasing function with $\lambda(0)=0$, such that for some $a>0$ and $s>s_{0}$

$$
\frac{\lambda(l)}{l} \geq a \frac{\lambda\left(l_{s+1}\right)}{l_{s+1}} \quad \text { for any } \quad l \in\left[l_{s+1}, l_{s}\right) .
$$

Then

$$
\liminf _{s \rightarrow \infty} N_{s} \lambda\left(l_{s}\right) \geq \mathscr{H}_{\lambda}(C) \geq \frac{a}{2} \liminf _{s \rightarrow \infty} N_{s} \lambda\left(l_{s}\right) .
$$

Two slightly more delicate results that we need, give estimates of the Hausdorff measure of (symmetric) Cantor sets and cylinder sets in higher dimensions. Note also that we are not interested in the exact value of the Hausdorff measure but only in its positivity.

Lemma B (Hatano, [7]). Let $h$ be a continuous increasing function with $h(0)=0$. Let $\left\{k_{q}\right\}_{q=1}^{\infty}$ be a sequence of positive integers and $\left\{l_{q}\right\}_{q=0}^{\infty}, l_{0}=1$ 
be a sequence of positive numbers that satisfy $k_{q+1} l_{q+1}<l_{q}$. The generalized symmetric Cantor set $E$ in $\mathrm{R}^{m}$ defined by the sequences $\left\{k_{q}\right\}$ and $\left\{l_{q}\right\}$ is constructed in the following way: Let $C_{0}=[0,1], C_{1}$ is obtained from $C_{0}$ by removing $k_{1}-1$ open intervals of equal lengths such that remaining $k_{1}$ closed intervals are of length $l_{1}$. Then, to get $C_{2}, k_{2}-1$ open intervals are removed from each interval of $C_{1}$ such that remaining intervals are of length $l_{2}$, etc. Define $C=\bigcap_{n} C_{n}$ and $E=C \times C \times \cdots \times C \subset[0,1]^{m}$.

Then $\mathscr{H}_{h}(E)>0$ if and only if $\liminf _{q \rightarrow \infty}\left(k_{1} \ldots k_{q}\right)^{m} h\left(l_{q}\right)>0$.

The measure used in [7] is not the classical Hausdorff measure but one defined using coverings by all open cubes. As we mentioned above, under our condition on $h$ the two measures are equivalent (up to a multiplicative constant).

The next statement is intuitively clear but we were not able to find a precise reference, so we outline a short proof.

Lemma 2.1. Let $h(t)=t^{k-1} v(t)$, where $v$ is an increasing continuous function on $[0,+\infty)$ and $v(0)=0$. Assume also that $v(t / 2) \geq c v(t)$ for some $c>0$. If $F \subset[0,1]$ is compact, $\mathscr{H}_{v}(F)>0$, and $E=F \times[0,1]^{k-1} \subset \mathrm{R}^{k}$, then $\mathscr{H}_{h}(E)>0$.

Proof. We will use that $\mathscr{H}_{v}$ is equivalent to $N_{v}$ and $\mathscr{H}_{h}$ is equivalent to $N_{h}$. Assume that $N_{h}(E)=0$, then for any $\epsilon>0$ and $\delta>0$ there exists a finite family of half-open dyadic cubes $\left\{Q_{\alpha}\right\}$ with sides $l_{\alpha}=2^{-n_{\alpha}}<\delta$ that covers $E=F \times[0,1]^{k-1}$ and such that $\sum_{\alpha} h\left(l_{\alpha}\right)<\epsilon$. Indeed we can find an infinite family for which $\sum h\left(l_{\alpha}\right)<2^{-k} \epsilon$, then for each cube $Q$ in this family, take an open cube that contains $Q$, has side length which is twice that of $Q$ and can be covered by $2^{k}$ half-open dyadic cubes of the same size as $Q$. Then we choose a finite sub-cover of the compact set $E$.

Let $n=\min _{\alpha} n_{\alpha}$ and $N=\max _{\alpha} n_{\alpha}$, we divide [0,1] into dyadic intervals $I_{j}$ of length $2^{-n},[0,1]=\bigcup_{j} I_{j}$. For each $j$ consider cubes $K_{j, s}=I_{j} \times J_{s}, 1 \leq$ $s \leq 2^{n(k-1)}$, where $J_{s}$ is a dyadic subcube of $[0,1]^{k-1}$ with side length $2^{-n}$. Now for each $s$ let

$$
d_{j, s}=\sum_{\alpha: Q_{\alpha} \subset K_{j, s}} h\left(l_{\alpha}\right) .
$$

Choose $t=t(j)$ such that $d_{j, t}=\min _{s} d_{j, s}$ and replace the covering $\left\{Q_{\alpha}\right\}$ by a new one $\left\{Q_{\beta}\right\}$ such that $\sum_{\beta} h\left(l_{\beta}\right) \leq \sum_{\alpha} h\left(l_{\alpha}\right)$, and for each $j$ the cubes $\left\{Q_{\beta}\right\}$ contained in $K_{j, s}$ can be obtained from the cubes $\left\{Q_{\alpha}\right\}$ contained in $K_{j, t(j)}$ by translation. If for the new family $\min n_{\beta}>n$, we repeat the procedure. If not, we get some chains of cubes $K_{j, 1}, \ldots, K_{j, 2^{(k-1) n}}$ in the new family and repeat the procedure on the complement of these chains. Anyway the size of 
the smallest cubes is always at least $2^{-N}$ and after finitely many steps we find a family of intervals $I_{\gamma}$ of length $l_{\gamma}<\delta$ that covers $F$ and

$$
\sum_{\gamma} v\left(l_{\gamma}\right)=\sum_{\gamma} h\left(l_{\gamma}\right) l_{\gamma}^{-(k-1)}<\epsilon .
$$

Thus $\mathscr{H}_{v}(F)=0$.

\section{Sets of extremal growth or decay}

\subsection{Lebesgue measure of sets of extremal growth}

In this subsection we first estimate the Lebesgue measure of the sets $E^{ \pm}(u)$ for arbitrary $u \in \Phi_{v}^{m}$.

Proposition 3.1. Suppose that $u \in \Phi_{v}^{m}$, then $\sigma\left(E^{-}(u)\right)=0$.

Proof. We have $E^{-}(u)=\bigcup_{n} F_{n}=\bigcup_{n}\left\{\zeta \in S: u(r \zeta) \leq-\frac{1}{n} v(r), r \geq\right.$ $\left.1-\frac{1}{n}\right\}$. Assume $\sigma\left(E^{-}(u)\right)>0$. Then $\sigma\left(F_{n}\right)>0$ for some $n$, and $F_{n}$ is a compact subset of $S$. Let $G=\bigcup_{\zeta \in F_{n}} \Gamma_{\zeta}^{a}$ and $G_{\alpha}=G \cap \alpha \mathrm{B}$ for $\alpha<1$. We have $\partial G=F_{n} \cup L$, where $L=\partial G \cap \mathrm{B}$.

We will estimate $u(0)$ using harmonic measure in the domain $G_{\alpha}$. First, it follows from Dahlberg's theorem that $\omega\left(0, F_{n}, G\right)=c>0$. Now let $L_{\alpha}=$ $\partial G \cap \alpha \mathrm{B}=L \cap \alpha \mathrm{B}$ and let $p_{\alpha}(A)$ be the radial projection of a set $A$ onto $\alpha S$, where $0<\alpha \leq 1$. Then

$$
\partial G_{\alpha}=L_{\alpha} \cup \alpha F_{n} \cup p_{\alpha}\left(L \backslash L_{\alpha}\right) .
$$

Choose $s>1-\frac{1}{n}$ such that

$$
\omega\left(0, L \backslash L_{s}, G\right)<\frac{c}{3 n} \quad \text { and } \quad \sigma\left(p_{1}\left(L \backslash L_{s}\right)\right)<\frac{\gamma_{m-1} c}{3 n} .
$$

Let $s<t<1$. Then, since $G_{t} \subset G$,

$$
\omega\left(0, L_{t} \backslash L_{s}, G_{t}\right) \leq \omega\left(0, L_{t} \backslash L_{s}, G\right) \leq \omega\left(0, L \backslash L_{s}, G\right)<\frac{c}{3 n} .
$$

Further,

$$
\omega\left(0, p_{t}\left(L \backslash L_{t}\right), G_{t}\right) \leq \omega\left(0, p_{t}\left(L \backslash L_{t}\right), t \mathrm{~B}\right)=\frac{1}{\gamma_{m-1}} \sigma\left(p_{1}\left(L \backslash L_{t}\right)\right)<\frac{c}{3 n} .
$$

Finally, we want to estimate $\omega\left(0, t F_{n}, G_{t}\right)$. Note that $t G \subset G_{t}$, then

$$
\omega\left(0, t F_{n}, G_{t}\right) \geq \omega\left(0, t F_{n}, t G\right)=\omega\left(0, F_{n}, G\right)=c .
$$


Now we apply the estimates for the function $u$, which is harmonic in $G_{t}$, using that $\partial G_{t}=L_{s} \cup\left(L_{t} \backslash L_{s}\right) \cup t F_{n} \cup p_{t}\left(L \backslash L_{t}\right)$.

$$
u(0) \leq v(s) \omega\left(0, L_{s}, G\right)+v(t) \frac{2 c}{3 n}-\frac{v(t)}{n} c \leq v(s)-\frac{v(t) c}{3 n} .
$$

When $t$ goes to 1 we get a contradiction, since $v(t) \rightarrow \infty$.

Thus the estimate from above for $u, u(x) \leq K v(|x|)$, implies that the set of the radii along which $u$ decays at least as fast as $-v$ has zero measure. Theorem 1.1 formulated in the introduction follows.

To deal with the set $E^{+}(u)$ for $u \in \Phi(v)$, we assume that the function $v$ fulfills (2). The proof follows the argument from [4].

Lemma 3.2. Let $u \in \Phi_{v}^{m}$ where $v$ satisfies (2) and assume $u(x)>c v(|x|)$ for some $x \in \mathrm{B}$. Then there exists $\tau=\tau(K, D, c)>0$ such that $u\left(x^{\prime}\right)>c / 2 v(|x|)$ whenever $\left|x-x^{\prime}\right|<\tau(1-|x|),\left|x^{\prime}\right|=|x|$.

The same statement holds if we write $<$ in both inequalities and assume that $c<0$.

Proof. Let $y, y^{\prime} \in \mathrm{B}$. Assume that $|y|=\left|y^{\prime}\right|$ and $\left|y-y^{\prime}\right|<\tau_{1}(1-|y|)$, where $\tau_{1}<1$, then for any $\zeta \in S$

$$
\left|y^{\prime}-\zeta\right| \geq|y-\zeta|-\left|y-y^{\prime}\right|>|y-\zeta|-\tau_{1}|y-\zeta|=\left(1-\tau_{1}\right)|y-\zeta| .
$$

Thus $|y-\zeta|^{m}\left(1-\tau_{1}\right)^{m}<\left|y^{\prime}-\zeta\right|^{m}$ and

$$
P(y, \zeta)>\left(1-\tau_{1}\right)^{m} P\left(y^{\prime}, \zeta\right) .
$$

Let $r=|x|, R=(1+r) / 2$ and denote $q=q\left(\tau_{1}\right)=\left(1-\tau_{1}\right)^{m}$. We apply (10) with $y=\frac{x}{R}, y^{\prime}=\frac{x^{\prime}}{R}$ and $\left|y-y^{\prime}\right|<\tau_{1}(1-|y|)$. Then

$$
\begin{aligned}
u(x) & =\int_{S} u(R \zeta) P\left(\frac{x}{R}, \zeta\right) d \sigma(\zeta) \\
& =q u\left(x^{\prime}\right)+\int_{S} u(R \zeta)\left(P\left(\frac{x}{R}, \zeta\right)-q P\left(\frac{x^{\prime}}{R}, \zeta\right)\right) d \sigma(\zeta) \\
& \leq q u\left(x^{\prime}\right)+\int_{S} K v(R)\left(P\left(\frac{x}{R}, \zeta\right)-q P\left(\frac{x^{\prime}}{R}, \zeta\right)\right) d \sigma(\zeta) \\
& =q u\left(x^{\prime}\right)+(1-q) K v(R) \leq q u\left(x^{\prime}\right)+(1-q) K D v(r) .
\end{aligned}
$$

If $\tau_{1}$ is such that $c-(1-q) K D \geq \frac{c}{2} q$ and $\left|x-x^{\prime}\right|<\frac{\tau_{1}}{2}(1-r)<\tau_{1}\left(1-\frac{r}{R}\right)$, then $u\left(x^{\prime}\right)>\frac{c}{2} v(r)$. To complete the proof it suffices to choose $\tau(K, D, c)=\tau_{1} / 2$. 
For the second case when $c<0$, we use the inequality

$$
u\left(x^{\prime}\right) \leq q u(x)+(1-q) K D v(r)<(q c+(1-q) K D) v(r)
$$

and choose $\tau_{1}$ such that $q c+(1-q) K D \leq \frac{c}{2}$.

COROLlary 3.3. If $u \in \Phi_{v}^{m}$ where $v$ satisfies (2), then $\sigma\left(E^{+}(u)\right)=0$.

Proof. Note that by Lemma $3.2 u$ is bounded from below in $\Gamma_{\zeta}^{a}$ for any $\zeta \in E^{+}(u)$ and some $a=a(\zeta)$. Then by results of L. Carleson [5] (see also [9], [6]), $u$ has a finite non-tangential limit at almost each point of $E^{+}(u)$. Applying the lemma once again, we see that the non-tangential limit at $\zeta \in E^{+}(u)$ is infinite. Thus $\sigma\left(E^{+}(u)\right)=0$.

\subsection{Estimates of Hausdorff measures}

For weights that satisfy the doubling condition we can give more precise estimates of the size of the exceptional sets. We now prove Theorem 1.2(a) formulated in the introduction.

Proof OF Theorem 1.2(a). We start with $E^{+}(u)$. It is enough to prove the statement for each set

$$
E_{n}=\left\{\zeta \in S: u(r \zeta) \geq \frac{1}{n} v(r), r \geq 1-\frac{1}{n}\right\} .
$$

By Lemma 3.2, there exists $a$ such that $u(x) \geq \frac{1}{2 n} v(|x|)$ for any $x \in \Gamma_{\zeta}^{a}$ where $|x|>1-\frac{1}{n}$ and $\zeta \in E^{+}(u)$.

Let $G=\bigcup_{\zeta \in E_{n}} \Gamma_{\zeta}^{a}$ and $G_{t}=G \cap t \mathrm{~B}$. Clearly we may assume that $u \geq c_{0}$ on $G$ for some $c_{0}<0$. Let $b=b(a)$ be such that

$$
\partial G_{t} \cap t S=t E_{n}^{b(1-t)}=\left\{t \zeta \in t S:\left|\zeta-\zeta_{0}\right|<b(1-t) \text { where } \zeta_{0} \in E_{n}\right\} .
$$

Then by harmonic measure estimate for $G_{t}$ when $t>1-\frac{1}{n}$, we obtain

$$
u(0) \geq c_{0}+\omega\left(0, t E_{n}^{b(1-t)}, G_{t}\right) \frac{v(t)}{2 n} .
$$

By Theorem A there exists $C$ and $\gamma>0$ that depend only on $a$ such that

$$
\omega\left(0, A, G_{t}\right) \geq C \sigma(A)^{\gamma},
$$

here $\sigma$ is the (m-1)-dimensional surface measure on $t S$. This implies

$$
\sigma\left(E_{n}^{b(1-t)}\right)^{\gamma} \leq \frac{C_{1}}{v(t)}
$$


where $C_{1}=C_{1}(n, u, a)$. So for all $\epsilon>0$ small enough we get by applying (2)

$$
\sigma\left(E_{n}^{\epsilon}\right) \leq C_{2}\left(v\left(1-\frac{\epsilon}{b}\right)\right)^{-1 / \gamma} \leq C_{3}(v(1-\epsilon))^{-1 / \gamma} .
$$

We cover $E_{n}$ by a finite collection of balls $\left\{B_{j}: j \in J\right\}$ of radius $\frac{\epsilon}{5}$ and centers at points in $E_{n}$. By the Vitali covering lemma (see for example [8, p. 2]) there exists a subcollection $J^{\prime} \subseteq J$ where $\left\{B_{j}: j \in J^{\prime}\right\}$ are disjoint and $\bigcup_{j \in J} B_{j} \subseteq \bigcup_{j \in J^{\prime}} 5 B_{j}$, and we also have $\bigcup_{j \in J^{\prime}} 5 B_{j} \subseteq E_{n}^{\epsilon}$. Then $E_{n}$ can be covered by $N_{\epsilon}$ balls $\left\{5 B_{j}: j \in J^{\prime}\right\}$ of radius $\epsilon$, where

$$
\epsilon^{m-1} N_{\epsilon} \leq 5^{m-1} \sigma\left(E_{n}^{\epsilon}\right),
$$

thus

$$
N_{\epsilon} \leq 5^{m-1} \epsilon^{-m+1} C_{3}(v(1-\epsilon))^{-1 / \gamma} .
$$

Then

$$
\mathscr{H}_{\lambda}\left(E_{n}\right) \leq \liminf _{\epsilon \rightarrow 0} N_{\epsilon} \lambda(\epsilon) \leq \liminf _{\epsilon \rightarrow 0} 5^{m-1} \epsilon^{-m+1} C_{3}(v(1-\epsilon))^{-1 / \gamma} \lambda(\epsilon) .
$$

Since $\lambda(t)=o\left(t^{m-1}(v(1-t))^{w}\right),(t \rightarrow 0)$, for any $w>0$, we get $\mathscr{H}_{\lambda}\left(E_{n}\right)=0$.

The proof for $\mathscr{H}_{\lambda}\left(E^{-}(u)\right)$ is similar; we then use the second part of Lemma 3.2.

Remark 3.4. If $v(t)=(1-t)^{-\gamma}$ for some $\gamma>0$ and $u \in \Phi_{v}^{m}$, then the theorem above implies in particular that $\mathscr{H}_{\lambda}\left(E^{+}(u)\right)=0$ and $\mathscr{H}_{\lambda}\left(E^{-}(u)\right)=0$ when $\lambda(t)=t^{m-1} \log \frac{1}{t}$. On the other hand, we will show in section 3.4 that for any $\epsilon>0$ there exists $u \in \Phi_{v}^{m}$ such that

$$
\operatorname{dim} E^{+}(u)>m-1-\epsilon .
$$

\subsection{Auxiliary functions}

We now begin to prove Theorem 1.2(b). First we construct auxiliary functions $u_{k}$ in B that resemble $\operatorname{Im}\left(z^{2^{k}}\right)$ in the unit disk.

For each positive integer $k$ let $S_{k}$ and $T_{k}$ be subsets of the interval $[0,2 \pi)$ defined by

$$
\begin{aligned}
S_{k} & =\bigcup_{j=0}^{2^{k}-1}\left[2 j \pi 2^{-k},(2 j+1) \pi 2^{-k}\right), \\
T_{k} & =\bigcup_{j=0}^{2^{k}-1}\left[(2 j+1 / 4) \pi 2^{-k},(2 j+3 / 4) \pi 2^{-k}\right] .
\end{aligned}
$$


Then on the unit sphere $S$ in $\mathrm{R}^{m}$ we define

$$
E_{k}=\left\{\eta \in S, \eta=\left(t \cos \phi, t \sin \phi, \eta_{3}, \ldots, \eta_{m}\right), t \geq 0, \phi \in S_{k}\right\},
$$

and

$$
F_{k}=\left\{\eta \in S, \eta=\left(t \cos \phi, t \sin \phi, \eta_{3}, \ldots, \eta_{m}\right), t \geq 3 / 4, \phi \in T_{k}\right\} .
$$

Let $f_{k}=1$ on $E_{k}$ and $f_{k}=-1$ on $S \backslash E_{k}$. Further, let $u_{k}=P * f_{k}$ be the corresponding harmonic function in the unit ball $\mathrm{B}$.

Lemma 3.5. The function $u_{k}$ has the following properties

(a) $\left|u_{k}\right| \leq 1$ on $\mathrm{B}$;

(b) $u_{k}(r \eta) \geq 0$ when $\eta \in E_{k}$;

(c) For each $d \in \mathrm{N}$ there exists $c_{d, m}$ such that $\left|u_{k}(x)\right| \leq c_{d, m} 2^{-k d}(1-|x|)^{-d}$;

(d) There exists $a_{m}>0$ such that $u_{k}(r \eta)>1 / 4$ when $r>1-a_{m} 2^{-k}$ and $\eta \in F_{k}$.

Proof. By the maximum principle (a) follows immediately. Note further that $f_{k}\left(x_{1}, x_{2}, \ldots, x_{m}\right)=-f_{k}\left(x_{1},-x_{2}, \ldots, x_{m}\right)$, and thus

$$
u_{k}(x)=\frac{1}{\gamma_{m-1}} \int_{S} \frac{1-|x|^{2}}{|x-y|^{m}} f_{k}(y) d y
$$

satisfies $u_{k}\left(x_{1}, x_{2}, \ldots, x_{m}\right)=-u_{k}\left(x_{1},-x_{2}, \ldots, x_{m}\right)$. In particular,

$$
u_{k}\left(x_{1}, 0, \ldots, x_{m}\right)=0 .
$$

Let $\alpha_{k}=\pi 2^{-k}$ and

$$
A_{k}=\left[\begin{array}{ccccc}
\cos \alpha_{k} & -\sin \alpha_{k} & 0 & \ldots & 0 \\
\sin \alpha_{k} & \cos \alpha_{k} & 0 & \ldots & 0 \\
0 & 0 & & & \\
\vdots & \vdots & & I_{m-2} & \\
0 & 0 & & &
\end{array}\right],
$$

where $I_{m-2}$ is the identity matrix. Then $A_{k}$ is an orthogonal matrix and the corresponding transformation of $\mathrm{R}^{m}$ maps the unit sphere to itself, moreover $A_{k}\left(E_{k}\right)=S \backslash E_{k}$. Then

$$
f_{k}\left(A_{k} x\right)=-f_{k}(x) \quad \text { and } \quad u_{k}\left(\left(A_{k}\right)^{-1} x\right)=-u_{k}(x) .
$$

Now, taking into account (11), we get

$$
u_{k}\left(s \cos l \alpha_{k}, s \sin l \alpha_{k}, x_{3}, \ldots, x_{m}\right)=0
$$


for any $l=0,1, \ldots, 2^{k+1}-1$. Fix $l$ and consider the set

$$
G_{k, l}=\left\{x \in \mathrm{B}, x=\left(s \cos \phi, s \sin \phi, x_{3}, \ldots, x_{m}\right), \phi \in\left(l \alpha_{k},(l+1) \alpha_{k}\right)\right\} .
$$

The boundary of $G_{k, l}$ consists of a part of the unit sphere and of subsets of the hyperplanes

$$
\left\{\left(\sin l \alpha_{k}\right) x_{1}-\left(\cos l \alpha_{k}\right) x_{2}=0\right\}
$$

and

$$
\left\{\left(\sin (l+1) \alpha_{k}\right) x_{1}-\left(\cos (l+1) \alpha_{k}\right) x_{2}=0\right\} .
$$

On both subsets of the hyperplanes $u_{k}=0$, and on the corresponding part of the sphere all boundary values of $u_{k}$ equal 1 if $l$ is even and -1 if $l$ is odd. Anyway, $u_{k}$ does not change sign in $G_{k, l}$ and (b) follows.

To prove (c) assume first that $d=1$. We write

$$
\begin{aligned}
u_{k}(x) & =\frac{1}{\gamma_{m-1}} \int_{E_{k}} \frac{1-|x|^{2}}{|x-y|^{m}} d y-\frac{1}{\gamma_{m-1}} \int_{S \backslash E_{k}} \frac{1-|x|^{2}}{|x-y|^{m}} d y \\
& =\frac{1}{\gamma_{m-1}} \int_{E_{k}}\left(\frac{1-|x|^{2}}{|x-y|^{m}}-\frac{1-|x|^{2}}{\left|x-A_{k} y\right|^{m}}\right) d y .
\end{aligned}
$$

We want to estimate the difference under the integral sign. Note that

$$
\max _{y \in \mathrm{B}}\left|y-A_{k} y\right|=2 \sin \alpha_{k} / 2<\alpha_{k}
$$

and assume that $1-|x|>\alpha_{k}$, then

$$
\begin{aligned}
\left|\frac{1}{|x-y|^{m}}-\frac{1}{\left|x-A_{k} y\right|^{m}}\right| & \leq \frac{m\left|y-A_{k} y\right|\left(\left|x-A_{k} y\right|+\alpha_{k}\right)^{m-1}}{|x-y|^{m}\left|x-A_{k} y\right|^{m}} \\
& \leq \frac{m \alpha_{k} 2^{m-1}}{(1-|x|)|x-y|^{m}} .
\end{aligned}
$$

We obtain $\left|u_{k}(x)\right| \leq c_{m} \pi 2^{-k}(1-|x|)^{-1}$ when $1-|x|>\alpha_{k}$, otherwise the inequality follows from (a).

In general, for $d \geq 2$, we define $V_{x}(y)=|x-y|^{-m}$ and notice that

$$
\begin{aligned}
& \int_{E_{k}} V_{x}\left(A_{k}^{j} y\right) d y=\int_{E_{k}} V_{x}(y) d y, \quad \text { when } j \text { is even, and } \\
& \int_{E_{k}} V_{x}\left(A_{k}^{j} y\right) d y=\int_{S \backslash E_{k}} V_{x}(y) d y, \quad \text { when } j \text { is odd. }
\end{aligned}
$$


Further, we have $\sum_{j=0}^{d}(-1)^{j}\left(\begin{array}{l}d \\ j\end{array}\right)=(1-1)^{d}=0$ and $\sum_{j=0}^{d}\left(\begin{array}{l}d \\ j\end{array}\right)=(1+1)^{d}=$ $2^{d}$. Thus

$$
\begin{aligned}
u_{k}(x) & =\frac{1-|x|^{2}}{\gamma_{m-1}} \int_{E_{k}} V_{x}(y) d y-\frac{1-|x|^{2}}{\gamma_{m-1}} \int_{S \backslash E_{k}} V_{x}(y) d y \\
& =\frac{1-|x|^{2}}{\gamma_{m-1}} 2^{-d+1} \int_{E_{k}} \sum_{j=0}^{d}(-1)^{j}\left(\begin{array}{l}
d \\
j
\end{array}\right) V_{x}\left(A_{k}^{j} y\right) d y .
\end{aligned}
$$

To estimate the sum under the integral sign let

$$
y=\left(y_{0} \cos \psi, y_{0} \sin \psi, y_{1}\right) \in \mathrm{R} \times \mathrm{R} \times \mathrm{R}^{m-2} .
$$

We have $A_{k}^{j} y=\left(y_{0} \cos \left(\psi+j \alpha_{k}\right), y_{0} \sin \left(\psi+j \alpha_{k}\right), y_{1}\right)$ and $V_{x}(y)=$ $h_{x, y_{0}, y_{1}}(\psi)$. Then we write the Taylor polynomial of order $d-1$ for $h_{x, y_{0}, y_{1}}$ at $\psi$,

$$
\begin{aligned}
V_{x}\left(A_{k}^{j} y\right) & =h_{x, y_{0}, y_{1}}\left(\psi+j \alpha_{k}\right) \\
& =\sum_{l=0}^{d-1} \frac{h_{x, y_{0}, y_{1}}^{(l)}(\psi)}{l !}\left(j \alpha_{k}\right)^{l}+\frac{h_{x, y_{0}, y_{1}}^{(d)}\left(\psi+\beta_{j k}\right)}{d !}\left(j \alpha_{k}\right)^{d} .
\end{aligned}
$$

A straightforward estimate of the derivatives of $h$ shows that

$$
\begin{aligned}
\left|h_{x, y_{0}, y_{1}}^{(d)}(\phi)\right| & \leq C\left|x-\left(y_{0} \cos \phi, y_{0} \sin \phi, y_{1}\right)\right|^{-m-d} \\
& \leq C\left(|x-y|-y_{0}|\phi-\psi|\right)^{-m-d},
\end{aligned}
$$

where $C$ depends only on $m$ and $d$. Applying the difference relation

$$
\sum_{j=0}^{d}(-1)^{j}\left(\begin{array}{l}
d \\
j
\end{array}\right) j^{l}=0
$$

when $l<d$ (see for example [18, p. 42]), we get

$$
\left|\sum_{j=0}^{d}(-1)^{j}\left(\begin{array}{l}
d \\
j
\end{array}\right) V_{x}\left(A_{k}^{j} y\right)\right| \leq C 2^{-d k}\left(|x-y|-d \alpha_{k}\right)^{-m-d} .
$$

Now for $1-|x|>2 d \alpha_{k}$ and $|y|=1$ we obtain

$$
\left|\sum_{j=0}^{d}(-1)^{j}\left(\begin{array}{l}
d \\
j
\end{array}\right) V_{x}\left(A_{k}^{j} y\right)\right| \leq C 2^{m+d} 2^{-d k}|x-y|^{-m}(1-|x|)^{-d},
$$


and the integration over $E_{k}$ gives

$$
u_{k}(x) \leq c_{d, m} 2^{-d k}(1-|x|)^{-d} .
$$

Note also that for $1-|x|<2 d \alpha_{k}$ the inequality above (with a large enough constant) follows from $\left|u_{k}\right| \leq 1$, so (c) holds.

Finally, we prove (d). Let $\eta \in F_{k}$ and $x=r \eta$. It is easy to check that $B\left(\eta, 2^{-k-1}\right) \subset E_{k}$. A direct calculation shows that for $a_{m}$ small enough

$$
\frac{1}{\gamma_{m-1}} \int_{S \backslash B\left(\eta, 2^{-k-1}\right)} \frac{1-r^{2}}{|r \eta-y|^{m}} d y<\frac{3}{8},
$$

when $r>1-a_{m} 2^{-k}$. Thus $u_{k}(x)>1-2 \frac{3}{8}=\frac{1}{4}$.

It will be more convenient to use functions like $\operatorname{Re}\left(z^{2^{k}}\right)$, so we define

$$
h_{k}(x)=u_{k}\left(A_{k+1} x\right) ; \quad B_{k}=\bigcup_{j=0}^{2^{k}-1}\left[(2 j-1 / 4) \pi 2^{-k},(2 j+1 / 4) \pi 2^{-k}\right] .
$$

It is easy to check that (d) implies $h_{k}(r \eta)>1 / 4$ when

and $r>1-a_{m} 2^{-k}$.

\subsection{Construction of $u \in \Psi_{v}^{m}$ with a large set of radial growth}

Now we can prove Theorem 1.2(b).

Proof of Theorem 1.2(b). First we construct $v_{\beta}(t)=O\left(t v(1-t)^{\beta}\right)$. The assumption on $v$ implies

$$
\frac{d}{2} v\left(1-\frac{d}{2}\right)^{\alpha} \leq \frac{d}{2} D^{\alpha} v(1-d)^{\alpha}<\frac{3}{4} d v(1-d)^{\alpha}
$$

when $\alpha \leq \alpha_{0}$.

For simplicity we define a new function $g$ such that $v(r)=g\left(\frac{1}{1-r}\right)$. We will keep this notation throughout the paper. Then (2) is equivalent to

$$
g(2 x) \leq D g(x) .
$$

We choose $\alpha \leq \min \left\{\alpha_{0}, \beta\right\}$, and define $\nu_{\beta}$ by

$$
v_{\beta}\left(\pi 2^{-n}\right)=\pi 2^{-n} v\left(1-2^{-n}\right)^{\alpha}=\pi 2^{-n} g\left(2^{n}\right)^{\alpha}, \quad n \geq 2,
$$


and $v_{\beta}$ is linear on $\left[\pi 2^{-n-1}, \pi 2^{-n}\right]$. Then $\lim _{t \rightarrow 0} v_{\beta}(t)=0$ and $v_{\beta}$ is continuous and increasing. For $t \in\left[\pi 2^{-n-1}, \pi 2^{-n}\right.$ ) we have

$$
\begin{aligned}
\frac{v_{\beta}(t)}{\operatorname{tg}\left(\frac{1}{t}\right)^{\beta}} & \leq \frac{\pi 2^{-n} g\left(2^{n}\right)^{\alpha}}{\pi 2^{-n-1} g\left(2^{n} / \pi\right)^{\beta}}=2 \frac{g\left(2^{n}\right)^{\alpha}}{g\left(2^{n} / \pi\right)^{\beta}} \\
& \leq 2 \frac{g\left(2^{n}\right)^{\alpha}}{g\left(2^{n-2}\right)^{\beta}} \leq 2 D^{2 \beta} \frac{g\left(2^{n}\right)^{\alpha}}{g\left(2^{n}\right)^{\beta}} \leq 2 D^{2 \beta}
\end{aligned}
$$

when $n \geq n_{0}$, so $v_{\beta}(t)=O\left(t v(1-t)^{\beta}\right)$ when $t \rightarrow 0$. We also define a new function $\lambda_{\beta}(t)=t^{m-2} v_{\beta}(t)$.

Fix $A_{1}>1$ and define $b_{1}=1$,

$$
b_{n+1}=\min \left\{l: g\left(2^{l}\right)>A_{1} g\left(2^{b_{n}}\right)\right\}, \quad n=2,3, \ldots
$$

By assumption, $g\left(2^{b_{n+1}}\right) \leq D g\left(2^{b_{n+1}-1}\right)$, and by the way the $b_{n}$ 's are defined, $g\left(2^{b_{n+1}-1}\right) \leq A_{1} g\left(2^{b_{n}}\right)$. Then

$$
g\left(2^{b_{n+1}}\right) \leq D A_{1} g\left(2^{b_{n}}\right)=A_{2} g\left(2^{b_{n}}\right) .
$$

Let

$$
u(x)=\sum_{n=1}^{\infty} g\left(2^{b_{n}}\right) h_{b_{n}}(x) .
$$

We want to check that $u$ converges uniformly on compact subsets of B and $u \in \Phi_{v}^{m}$. Since $g$ fulfills (13), there exists $\gamma$ such that

$$
\frac{g\left(2^{l_{2}}\right)}{g\left(2^{l_{1}}\right)} \leq 2^{\gamma\left(l_{2}-l_{1}\right)}
$$

for $l_{1}, l_{2} \in \mathrm{N}$, just let $\gamma=\log _{2} D$. Choose $d>\gamma$ and note that (15) implies

$$
\frac{g\left(2^{b_{n+1}}\right) 2^{-b_{n+1} d}}{g\left(2^{b_{n}}\right) 2^{-b_{n} d}} \leq 2^{-(d-\gamma)\left(b_{n+1}-b_{n}\right)} \leq 2^{-(d-\gamma)},
$$

when $n>n_{0}$. Assume that $1-2^{-b_{N}}<|x|<1-2^{-b_{N+1}}$, then by Lemma 3.5(a) and (c),

$$
|u(x)| \leq \sum_{n=1}^{N} g\left(2^{b_{n}}\right)+c_{d, m} \sum_{n=N+1}^{\infty} g\left(2^{b_{n}}\right) 2^{-b_{n} d}(1-|x|)^{-d} .
$$

The first sum is bounded by $C_{1} g\left(2^{b_{N}}\right)$, and for $N$ large enough (16) implies that the second sum is bounded by $C_{2} g\left(2^{b_{N+1}}\right) 2^{-b_{N+1} d}(1-|x|)^{-d} \leq C_{2} g\left(2^{b_{N+1}}\right)$. 
Then by (14)

$$
|u(x)| \leq C_{3} g\left(2^{b_{N+1}}\right) \leq C_{4} g\left(2^{b_{N}}\right) \leq C_{4} g\left(\frac{1}{1-|x|}\right) .
$$

Finally, we show that $F=\bigcap_{n} H_{b_{n}} \subset E^{+}(u)$, where $H_{k}$ are defined by (12). Let $x=|x| \eta, \eta \in F \subset S$, and $1-a_{m} 2^{-b_{N}}<|x| \leq 1-a_{m} 2^{-b_{N+1}}$, where $a_{m}$ is as in Lemma 3.5; we may assume also that $x_{1}^{2}+x_{2}^{2}>1 / 4$. Then by (b) and (d) in Lemma 3.5 (see also the definition of $h_{k}$ above), we obtain

$$
u(x)=\sum_{n=1}^{\infty} g\left(2^{b_{n}}\right) h_{b_{n}}(x) \geq g\left(2^{b_{N}}\right) h_{b_{N}}(x) \geq \frac{1}{4} g\left(2^{b_{N}}\right) \geq C_{5} g\left(\frac{1}{1-|x|}\right) .
$$

Let $C=\bigcap_{n} B_{b_{n}} \subset[0,2 \pi)$ and $C_{j}=\bigcap_{n=1}^{j} B_{b_{n}}$. Then $C_{j}$ is a union of $N_{j}$ intervals of length $l_{j}=\frac{\pi}{4} 2^{-b_{j}}$, where some of the intervals are next to each other, and $C$ is a set as in Lemma A. Intervals of length $l_{j}$ are called intervals from $j$-th generation. Each of them contains $k_{j+1}$ intervals from the next generation. It is easy to show that $k_{j+1}=1$ if $b_{j+1}-b_{j}=1$, and $k_{j+1}=\frac{1}{4} 2^{b_{j+1}-b_{j}}$ if $b_{j+1}-b_{j}>1$. So $k_{j+1} \geq \frac{1}{4} 2^{b_{j+1}-b_{j}}$ and $N_{j} \geq\left(\frac{1}{4}\right)^{j} 2^{b_{j}}$.

Let $0<l \leq \frac{\pi}{4}$ and pick $t$ and $j$ in N such that $l_{j} \geq \frac{\pi}{4} 2^{-t} \geq l \geq \frac{\pi}{4} 2^{-t-1} \geq$ $l_{j+1}$. Then

$$
\begin{aligned}
\frac{v_{\beta}(l)}{l} & \geq \frac{v_{\beta}\left(\frac{\pi}{4} 2^{-t-1}\right)}{\frac{\pi}{4} 2^{-t}}=\frac{\pi 2^{-t-3} g\left(2^{t+3}\right)^{\alpha}}{\pi 2^{-t-2}} \geq \frac{1}{2} g\left(2^{b_{j}+3}\right)^{\alpha} \geq \frac{1}{2} g\left(2^{b_{j}}\right)^{\alpha} \\
& \geq \frac{1}{2 A_{2}^{\alpha}} g\left(2^{b_{j+1}}\right)^{\alpha} \geq \frac{1}{2 A_{2}^{\alpha} D^{2 \alpha}} g\left(2^{b_{j+1}} 4\right)^{\alpha}=\frac{1}{2 A_{2}^{\alpha} D^{2 \alpha}} \frac{v_{\beta}\left(l_{j+1}\right)}{l_{j+1}} .
\end{aligned}
$$

Lemma A with $v_{\beta}$ defined as above and $a=\frac{1}{2 A_{2}^{\alpha} D^{2 \alpha}}$ now yields

$$
\begin{aligned}
\mathscr{H}_{\nu_{\beta}}(C) & \geq \frac{a}{2} \liminf _{j \rightarrow \infty} N_{j} v_{\beta}\left(l_{j}\right) \geq \frac{a}{2} \liminf _{j \rightarrow \infty}\left(\frac{1}{4}\right)^{j} 2^{b_{j}} v_{\beta}\left(\frac{\pi}{4} 2^{-b_{j}}\right) \\
& =\frac{a \pi}{8} \liminf _{j \rightarrow \infty}\left(\frac{1}{4}\right)^{j} g\left(2^{b_{j}+2}\right)^{\alpha} \geq \frac{a \pi}{8} \liminf _{j \rightarrow \infty}\left(\frac{1}{4}\right)^{j} A_{1}^{j \alpha} g\left(2^{b_{0}}\right)^{\alpha} .
\end{aligned}
$$

By choosing $A_{1}^{\alpha}>4$ we obtain $\mathscr{H}_{\nu_{\beta}}(C)=\infty$.

Then by Lemma 2.1 for $\lambda_{\beta}(t)=t^{m-2} v_{\beta}(t)$ and the remark on the behavior of the Hausdorff measure under the Lipschitz map, we have

$$
\mathscr{H}_{\lambda_{\beta}}\left(E^{+}(u)\right) \geq \mathscr{H}_{\lambda_{\beta}}(F)>0 .
$$




\section{Positive harmonic functions}

\subsection{Proof of the Theorem 1.4}

We will now consider extremal growth on subsets of radii of the unit ball in $\mathrm{R}^{m}$ for positive functions. Let $v$ be a positive increasing continuous function on $[0,1)$ and assume $\lambda(t)=t^{m-1} v(1-t)$ is increasing. Let $u$ be a positive harmonic function on B and let $F_{v}^{+}(u)$ be defined by (3). For positive $u \in \Psi_{v}^{m}$, clearly $E^{+}(u) \subset F_{v}^{+}(u)$. Theorem 1.4 is a generalization of Theorem 2 in [4], where the result is proved for $v(r)=\log \left(\frac{1}{1-r}\right)$ and $m=2$. Note that we no longer assume that $u \in \Psi_{v}^{m}$.

The proof of Theorem 1.4 is similar to the one in [4], but the proof of Lemma 4.1 is new.

Let

$$
F_{n}=\left\{\zeta \in S: \limsup _{r \rightarrow 1} \frac{u(r \zeta)}{v(r)} \geq \frac{2}{n}\right\} .
$$

It suffices to prove that $\mathscr{H}_{\lambda}\left(F_{n}\right)<\infty$ for all $n$.

Clearly $u=P * \mu$ for some positive Borel measure $\mu$ on $S$. Let $h: S \rightarrow$ $[0, \pi]$ be given by $h\left(\cos \phi, \zeta^{\prime}\right)=\phi$ and define a measure on $[0, \pi]$ by $v=h_{*} \mu$, which means that $v(A)=\mu\left(h^{-1}(A)\right)$ for any measurable set $A \subset[0, \pi]$. The formula

$$
\int_{0}^{\pi} f(\psi) d v(\psi)=\int_{0}^{\pi} f^{\prime}(\phi) \nu((\phi, \pi]) d \phi
$$

is valid for $f \in C^{1}[0, \pi]$ that is non-decreasing and fulfills $f(0)=0$ and $f(t)>0$ for $t>0$ (see for example [17, p. 84]). By using it with $f(\phi)=$ $\tilde{P}_{m, r}(0)-\tilde{P}_{m, r}(\phi)$, we get the following integration by parts on $S$

$$
\begin{aligned}
\int_{S} P(r x, \zeta) d \mu(\zeta) & =\int_{S} \tilde{P}_{m, r}(h(\zeta)) d \mu(\zeta)=\int_{0}^{\pi} \tilde{P}_{m, r}(\phi) d v \\
& =-\int_{0}^{\pi}\left(\tilde{P}_{m, r}(0)-\tilde{P}_{m, r}(\phi)\right) d v+\tilde{P}_{m, r}(0) v([0, \pi]) \\
& =-\int_{0}^{\pi} Q_{m}(r, \phi) v((\phi, \pi]) d \phi+\tilde{P}_{m, r}(0) \mu(S) \\
& =\tilde{P}_{m, r}(\pi) \mu(S)+\int_{0}^{\pi} Q_{m}(r, \pi) v((0, \phi]) d \phi
\end{aligned}
$$

thus

$$
\int_{S} P(r x, \zeta) d \mu(\zeta)=\tilde{P}_{m, r}(\pi) \mu(S)+\int_{0}^{\pi} Q_{m}(r, \phi) \mu(\bar{B}(x, \phi)) d \phi .
$$

We need the following lemma: 
Lemma 4.1. For each $n$ there exists $k=k(m, n)>0$ such that for any $x \in F_{n}$ there is a decreasing sequence $\left\{\Delta_{j}\right\}, \Delta_{j} \rightarrow 0$ as $j \rightarrow \infty$, which satisfies

$$
\mu\left(B\left(x, \Delta_{j}\right)\right) \geq k \sigma\left(B\left(x, \Delta_{j}\right)\right) v\left(1-\Delta_{j}\right) .
$$

Suppose this lemma is already proved. Let $K$ be a compact subset of $F_{n}$ and let $B_{j}=B\left(x_{j}, a_{j}\right)$, where $x_{j} \in K$ and $a_{j}<\epsilon$. For each $\epsilon>0$ we can cover $K$ with a finite collection of such balls $\left\{B_{j}: j \in J\right\}$ which satisfy $\mu\left(B_{j}\right) \geq k \sigma\left(B_{j}\right) v\left(1-a_{j}\right)$. By the Vitali covering lemma (see for example [8, p. 2]) there exists a subcollection $J^{\prime} \subseteq J$ where $\left\{B_{j}: j \in J^{\prime}\right\}$ are disjoint and $\bigcup_{j \in J} B_{j} \subseteq \bigcup_{j \in J^{\prime}} 5 B_{j}$. Using (4) and Lemma 4.1 we obtain

$$
\begin{aligned}
\sum_{j \in J^{\prime}} \lambda\left(5 a_{j}\right) & \leq C \sum_{j \in J^{\prime}} \sigma\left(B\left(x_{j}, 5 a_{j}\right)\right) v\left(1-a_{j}\right) \leq C 5^{m-1} \sum_{j \in J^{\prime}} \sigma\left(B_{j}\right) v\left(1-a_{j}\right) \\
& \leq C \frac{5^{m-1}}{k} \sum_{j} \mu\left(B_{j}\right) \leq C \frac{5^{m-1}}{k} \mu(S),
\end{aligned}
$$

which yields $\mathscr{H}_{\lambda}(K) \leq C \frac{5^{m-1}}{k} \mu(S)$. Thus $\mathscr{H}_{\lambda}\left(F_{n}\right) \leq C \frac{5^{m-1}}{k} \mu(S)<\infty$.

Proof of Lemma 4.1. Assume that $x=(1,0, \ldots, 0)$. Then by (17) and (6),

$$
\begin{aligned}
u(r x) & =\int_{S} P(r x, \zeta) d \mu(\zeta) \leq \mu(S)+\int_{0}^{\pi} \mu(B(x, \phi)) Q_{m}(r, \phi) d \phi \\
& \leq \mu(S)+\int_{0}^{d} \mu(B(x, \phi)) Q_{m}(r, \phi) d \phi+\mu(S) \int_{d}^{\pi} Q_{m}(r, \phi) d \phi \\
& \leq \mu(S)+\int_{0}^{d} \mu(B(x, \phi)) Q_{m}(r, \phi) d \phi+\mu(S) C_{6} d^{-m}
\end{aligned}
$$

Let $k<\left[\left(C_{2} C_{4}+C_{9}\right) 3 n\right]^{-1}$, where the constants are from (4), (5) and (7). For $x \in F_{n}$ there exists a sequence $\left\{r_{j}\right\}_{1}^{\infty}$ such that $r_{j} \nearrow 1$ and $u\left(r_{j}\right)>\frac{1}{n} v\left(r_{j}\right)$. We may assume that $r_{j}>\frac{1}{2}$. Now choose $d_{j}=d_{j}\left(r_{j}, v\right) \geq 1-r_{j}$ such that

$$
C d_{j}^{-m}<\frac{1}{3 n} v\left(r_{j}\right)
$$

and $d_{j} \rightarrow 0$ when $j \rightarrow \infty$. For $j>j_{0}, \mu(S)<\frac{1}{3 n} v\left(r_{j}\right)$. Then

$$
\int_{0}^{d_{j}} \mu(B(x, \phi)) Q_{m}\left(r_{j}, \phi\right) d \phi>\frac{1}{3 n} v\left(r_{j}\right) .
$$


We claim that this implies that for any $j$ there exists $\Delta_{j} \in\left(0, d_{j}\right)$ such that

$$
\mu\left(B\left(x, \Delta_{j}\right)\right) \geq k \sigma\left(B\left(x, \Delta_{j}\right)\right) v\left(1-\Delta_{j}\right),
$$

and the lemma follows. If not, there exists $j$ such that

$$
\mu(B(x, \phi))<k \sigma(B(x, \phi)) v(1-\phi)
$$

for any $\phi \in\left(0, d_{j}\right)$. Using (4) and the fact that $t^{m-1} v(1-t)$ is increasing, and then applying (5) and (7), we obtain

$$
\begin{aligned}
\int_{0}^{d_{j}} \mu(B(x, \phi)) Q_{m}\left(r_{j}, \phi\right) d \phi \\
<k \int_{0}^{d_{j}} \sigma(B(x, \phi)) v(1-\phi) Q_{m}\left(r_{j}, \phi\right) d \phi \\
\leq k C_{2}\left(1-r_{j}\right)^{m-1} v\left(r_{j}\right) \int_{0}^{1-r_{j}} Q_{m}\left(r_{j}, \phi\right) d \phi \\
\quad+k v\left(r_{j}\right) \int_{1-r_{j}}^{\pi} \sigma(B(x, \phi)) Q_{m}\left(r_{j}, \phi\right) d \phi \\
\quad \leq k\left(C_{2}\left(1-r_{j}\right)^{m-1} v\left(r_{j}\right) C_{4} \frac{1}{\left(1-r_{j}\right)^{m-1}}+C_{9} v\left(r_{j}\right)\right)<\frac{1}{3 n} v\left(r_{j}\right),
\end{aligned}
$$

and we have a contradiction.

CoROllaRy 4.2. If $v(r)=\left(\frac{1}{1-r}\right)^{\gamma}$ for $0 \leq \gamma<m-1$, then for any positive harmonic function $u$, the Hausdorff dimension of $F_{v}^{+}(u)$ is less than or equal to $m-1-\gamma$.

We will show in section 4.3 that this estimate is sharp, i.e. there exists $u$ such that

$$
\operatorname{dim} F_{v}^{+}(u)=m-1-\gamma .
$$

4.2. Measures that correspond to positive functions in $\Psi_{v}^{m}$

Let

$$
\Theta_{v}^{m}=\{u: \mathrm{B} \rightarrow \mathrm{R}, \Delta u=0,0<u(x) \leq K v(|x|)\} .
$$

We want to characterize all functions in $\Theta_{v}^{m}$ by their corresponding measure on $S$.

Proposition 4.3. Suppose $v$ satisfies (2) and let $u(x)=\int_{S} P(x, \zeta) d \mu(\zeta)$ where $\mu$ is a positive Borel measure on $S$. Then $u \in \Theta_{v}^{m}$ if and only if

$$
\mu(B(x, \phi)) \leq C \sigma(B(x, \phi)) g\left(\frac{\pi}{\phi}\right)
$$

for each ball $B(x, \phi) \subset S$. 
Proof. Assume $u \in \Theta_{v}^{m}$ and let $x \in S$. Then

$$
\begin{aligned}
v\left(1-\frac{\phi}{\pi}\right) & \geq \frac{1}{K} u\left(\left(1-\frac{\phi}{\pi}\right) x\right)=\frac{1}{K \gamma_{m-1}} \int_{S} \frac{1-\left(1-\frac{\phi}{\pi}\right)^{2}}{\left|x\left(1-\frac{\phi}{\pi}\right)-\zeta\right|^{m}} d \mu(\zeta) \\
& \geq \frac{1}{K \pi \gamma_{m-1}} \int_{B(x, \phi)} \frac{2 \phi-\frac{\phi^{2}}{\pi}}{(2 \phi)^{m}} d \mu(\zeta) \geq \frac{1}{K \pi \gamma_{m-1}} \frac{\mu(B(x, \phi))}{2^{m} \phi^{m-1}} \\
& \geq C \mu(B(x, \phi)) \frac{1}{\sigma(B(x, \phi))},
\end{aligned}
$$

thus $\mu(B(x, \phi)) \leq C \sigma(B(x, \phi)) g\left(\frac{\pi}{\phi}\right)$.

Conversely, suppose that (19) is fulfilled. Without loss of generality we may assume that $x=(1,0, \ldots, 0)$. Then by (17),

$$
\begin{aligned}
u(r x)= & \int_{S} P(r x, \zeta) d \mu(\zeta) \leq \mu(S)+\int_{0}^{\pi} \mu(B(x, \phi)) Q_{m}(r, \phi) d \phi \\
\leq & \mu(S)+\int_{0}^{1-r} \mu(B(x, \phi)) Q_{m}(r, \phi) d \phi \\
& \quad+\int_{1-r}^{\pi} \mu(B(x, \phi)) Q_{m}(r, \phi) d \phi \\
\leq & \mu(S)+\mu(B(x, 1-r)) \int_{0}^{1-r} Q_{m}(r, \phi) d \phi \\
& \quad+C \int_{1-r}^{\pi} \sigma(B(x, \phi)) g\left(\frac{\pi}{\phi}\right) Q_{m}(r, \phi) d \phi \\
\leq & \mu(S)+C \sigma(B(x, 1-r)) g\left(\frac{\pi}{1-r}\right) \int_{0}^{1-r} Q_{m}(r, \phi) d \phi \\
& \quad+C g\left(\frac{\pi}{1-r}\right) \int_{1-r}^{\pi} \sigma(B(x, \phi)) Q_{m}(r, \phi) d \phi .
\end{aligned}
$$

Furthermore, by (5) and (7), $u(r x) \leq \tilde{C} g\left(\frac{\pi}{1-r}\right) \leq \tilde{C}_{1} v(r)$.

\subsection{Proof of Theorem 1.3}

First, note that $v$ satisfies (2), in fact

$$
v\left(1-\frac{t}{2}\right)=\frac{2^{m-1} \lambda\left(\frac{t}{2}\right)}{t^{m-1}}<\frac{2^{m-1} \lambda(t)}{t^{m-1}}=2^{m-1} v(1-t) .
$$


Consider the set $A=[0, \pi] \times \ldots \times[0, \pi] \times[0,2 \pi]$ and the hyperspherical coordinates on $S$, i.e., we consider the function $f: A \rightarrow S$ defined by

$$
f\left(\phi_{1}, \ldots, \phi_{m-1}\right)=\left(\cos \phi_{1}, \sin \phi_{1} \cos \phi_{2}, \ldots, \sin \phi_{1} \ldots \sin \phi_{m-1}\right) .
$$

This function is bilipschitz on $[1,2]^{m-1}$. We will use a Cantor-type construction to get a set $C \subset[1,2]^{m-1} \subset A$. We first construct a set in $[1,2]$.

Let $F_{0}=[1,2]$. Define $d_{k}$ as

$$
d_{k}=\min \left\{n \in \mathrm{N}: g\left(2^{n}\right) \geq 2^{(m-1) k}\right\}, \quad k=2,3, \ldots
$$

Then $d_{k+1}>d_{k}$ for all $k$. By (13) we also have

$$
\frac{g\left(2^{d_{k}}\right)}{g\left(2^{d_{k-1}}\right)} \leq \frac{D g\left(2^{d_{k}-1}\right)}{g\left(2^{d_{k-1}}\right)} \leq \frac{D 2^{(m-1) k}}{2^{(m-1)(k-1)}}=D 2^{m-1}=\delta_{m} .
$$

We construct by induction sets $F_{k} \subset F_{k-1}$ such that $F_{k}$ consists of $n_{k}=2^{d_{k}-k}$ closed intervals of length $2^{-d_{k}}$ each. To obtain $F_{k}$ we divide each of the intervals of $F_{k-1}$ into $2^{d_{k}-d_{k-1}}$ equal subintervals and choose each second of them for $F_{k}$. Now let $C_{k}=F_{k} \times \cdots \times F_{k} \subset[1,2]^{m-1}$. The number of squares in $C_{k}$ is $N_{k}=2^{\left(d_{k}-k\right)(m-1)}$. Let also $C=\cap C_{k}$.

Let $v_{k}$ be the measures defined by $d v_{k}=2^{(m-1) k} \chi\left(C_{k}\right) d y$ on $[1,2]^{m-1}$, where $\chi\left(C_{k}\right)$ is the characteristic function of $C_{k}$. We also define the measures $\mu_{k}=f_{*} v_{k}$ on $S$. Denote $G_{k}=f\left(C_{k}\right)$ and $G=f(C)$, clearly $G=\bigcap G_{k}$.

Lemma 4.4. The sequence $\left\{\mu_{k}\right\}$ converges $*$-weakly to a measure $\mu$ and $u=P * \mu \in \Theta_{v}^{m}$.

Proof. The $*$-weak convergence of $\left\{\mu_{k}\right\}$ follows from the $*$-weak convergence of $\left\{v_{k}\right\}$, which we will prove now. Note that $v_{k}\left(C_{0}\right)=1$ for each $k$. Let $\left\{J_{i}\right\}_{i=1}^{N_{k}}$ be the squares of $C_{k}$. For each square $J_{i}$ the limit $v_{k}\left(J_{i}\right)$ as $k \rightarrow \infty$ exists because all values $v_{k}\left(J_{i}\right)$ are the same when $k>s$. For squares in $S \backslash C_{k}$ the limit will be 0 . Now each continuous function on [1,2] ${ }^{m-1}$ can be uniformly approximated by linear combinations of characteristic functions of small squares. Thus for each continuous function $f$ on $[1,2]^{m-1}$ there exists

$$
\lim _{k \rightarrow \infty} \int_{[1,2]^{m-1}} f d v_{k}
$$

and $v_{k}$ converge weakly to some positive measure $v$.

By Proposition 4.3 it suffices to check that

$$
\mu(B(x, r)) \leq C \sigma(B(x, r)) g\left(\frac{\pi}{r}\right)
$$


for each ball $B(x, r) \subset S$, in order to prove that $u=P * \mu \in \Theta_{v}^{m}$. This is true if a similar estimate is true for $v$.

Let $y \in[1,2]^{m-1}$ and let $B_{e}(y, r)$ be a Euclidean ball. Choose $s$ such that $2^{-d_{s}}<r \leq 2^{-d_{s-1}}$. Now take a square $Q \supset B_{e}(y, r)$ that is a union of dyadic cubes with side lengths $2^{-d_{s}}$, and let the side length of $Q$ be $2^{-d_{s}} l$ for some $l \in \mathrm{N}$ such that $2^{-d_{s}} l<4 r$. Then $|Q|<A\left|B_{e}(y, r)\right|$ where $A=A(m)$. By using (20), we obtain

$$
\begin{aligned}
v_{k}\left(B_{e}(y, r)\right) & \leq v_{k}(Q)=v_{s}(Q) \leq 2^{(m-1) s}|Q|<A\left|B_{e}(y, r)\right| g\left(2^{d_{s}}\right) \\
& \leq A \delta_{m}\left|B_{e}(y, r)\right| g\left(2^{d_{s-1}}\right) \leq A \delta_{m}\left|B_{e}(y, r)\right| g\left(\frac{1}{r}\right)
\end{aligned}
$$

which is the desired inequality.

To finish the proof of Theorem 1.3 we will show that $G \subset E^{+}(v)$ and $\mathscr{H}_{\lambda}(G)>0$. We have

$$
u(r x)=\int_{S} P(r x, \zeta) d \mu(\zeta) \geq \int_{0}^{\pi} \mu(B(x, \phi)) Q_{m}(r, \phi) d \phi .
$$

Let $x \in \bigcap G_{k}=G$ and $r \in[0,1)$. Choose $k_{0}$ such that $2^{(m-1)\left(k_{0}-1\right)} \leq$ $g\left(\frac{1}{1-r}\right)<2^{(m-1) k_{0}}$. Then it is not difficult to see that $\mu(B(x, \phi)) \geq$ $c 2^{(m-1) k_{0}} \phi^{m-1}$ for $\phi<1-r$ and

$$
\begin{aligned}
u(r x) & \geq \int_{0}^{1-r} \mu(B(x, \phi)) Q_{m}(r x, \phi) d \phi \\
& \geq c 2^{(m-1) k_{0}} \int_{0}^{1-r} \phi^{m-1} Q_{m}(r x, \phi) d \phi \geq c_{1} 2^{(m-1) k_{0}} \geq c_{1} g\left(\frac{1}{1-r}\right),
\end{aligned}
$$

when $r>r_{0}$. Thus $G=\bigcap G_{k} \subset E^{+}(u)$.

Finally we use Lemma B to estimate $\mathscr{H}_{\lambda}(C)$. We have

$$
\liminf _{k \rightarrow \infty} N_{k} \lambda\left(l_{k}\right)=\liminf _{k \rightarrow \infty} 2^{d_{k}-k} 2^{-d_{k}} g\left(2^{d_{k}}\right) \geq \liminf _{k \rightarrow \infty} \pi 2^{-k+1} 2^{k}=c>0 .
$$

Then $\mathscr{H}_{\lambda}(C)>0$ and $\mathscr{H}_{\lambda}(G) \geq c_{1} \mathscr{H}_{\lambda}(C)>0$ since $C=f^{-1}(G)$ and $f^{-1}$ is Lipschitz. We use also that

$$
\lambda\left(\frac{t}{2}\right)=\left(\frac{t}{2}\right)^{m-1} v\left(1-\frac{t}{2}\right) \geq t^{m-1} v(1-t) 2^{-m+1}=2^{-m+1} \lambda(t),
$$

see Section 2.3. 


\section{REFERENCES}

1. Bañuelos, R., and Moore, Ch. N., Probabilistic Behaviour of Harmonic Functions, Birkhäuser, Basel 1999.

2. Borichev, A., and Hedenmalm, H., Harmonic functions of maximal growth: invertibility and cyclicity in Bergman spaces, J. Amer. Math. Soc. 10 (1997), 761-796.

3. Borichev, A., and Lyubarskii, Yu., Uniqueness theorems for Korenblum type spaces, J. Anal. Math. 103 (2007), 307-329.

4. Borichev, A., Lyubarskiı̆, Yu., Malinnikova, E., and Thomas, P., Radial growth of functions in Korenblum space, Algebra i Analiz 21:6 (2009), 47-65.

5. Carleson, L., On the existence of boundary values for harmonic functions in several variables, Ark. Mat. 4 (1962), 393-399.

6. Dahlberg, B. E. J., Estimates of harmonic measure, Arch. Ration. Mech. Anal. 65 (1977), 275-288.

7. Hatano, K., Evaluation of Hausdorff measures of generalized Cantor sets, J. Sci. Hiroshima (A-I) 32 (1968), 371-379.

8. Heinonen, J., Lectures on Analysis on Metric Spaces, Springer, New York 2001.

9. Hunt, R. A., and Wheeden, R. L., On the boundary values of harmonic functions, Trans. Amer. Math. Soc. 132 (1968), 307-322.

10. Jerison, D. S., and Kenig, C. E., Boundary value problems on Lipschitz domains, pp. 1-68 in: Studies in Partial Differential Equations, MAA Stud. Math. 23, Math. Assoc. America, Washington 1982.

11. Kahane, J.-P., and Katznelson, Y., Sur le comportement radial des fonctions analytiques, C. R. Acad. Sci. Paris (A-B) 272 (1971), A718-A719.

12. Korenblum, B., An extension of the Nevanlinna theory, Acta Math. 135 (1975), 187-219.

13. Lyubarskii, Yu., and Malinnikova, E., Radial oscillation of harmonic functions in the Korenblum space, Bull. London Math. Soc. 44 (2012), 68-84.

14. Mattila, P., Geometry of Sets and Measures in Euclidean Spaces. Fractals and Rectifiability, Cambridge Studies in Adv. Math. 44, Cambridge Univ. Press., Cambridge 1995.

15. Privalov, I. I., Boundary properties of analytic functions (Russian), Gosudarstv. Izdat. Tehn.Teor. Lit., Moscow 1950.

16. Seip, K., An extension of the Blaschke condition, J. Lond. Math. Soc. (2) 51 (1995), 545-558.

17. Stroock, D. W., A concise introduction to the theory of integration, 3rd ed., Birkhäuser, Boston 1999.

18. Vilenkin, N. Ya., Combinatorics, Academic Press, New York 1971.

DEPARTMENT OF MATHEMATICAL SCIENCES

NORWEGIAN UNIVERSITY OF SCIENCE AND TECHNOLOGY

NO-7491 TRONDHEIM

NORWAY

E-mail: kjerstei@math.ntnu.no

eugenia@math.ntnu.no 